\title{
Cardiac resynchronization therapy with wireless left ventricular endocardial pacing: is this the direction to go?
}

\author{
Tomas Holubec, Julius Beckers, Zdenka Holubcova, Veronika Walter, Thomas Walther \\ Department of Thoracic and Cardiovascular Surgery, University Hospital Frankfurt and Johann Wolfgang Goethe University Frankfurt, Frankfurt, \\ Germany \\ Correspondence to: Tomas Holubec, MD, PhD. Department of Thoracic and Cardiovascular Surgery, University Hospital and Johann Wolfgang \\ Goethe University, Frankfurt, Germany. Email: tomasholubec@email.cz. \\ Provenance: This is an invited Editorial commissioned by the Section Editor Yue Liu (The First Affiliated Hospital, Harbin Medical University, \\ Harbin, China). \\ Comment on: Reddy VY, Miller MA, Neuzil P, et al. Cardiac Resynchronization Therapy With Wireless Left Ventricular Endocardial Pacing: The \\ SELECT-LV Study. J Am Coll Cardiol 2017;69:2119-29.
}

Submitted Mar 06, 2018. Accepted for publication May 07, 2018.

doi: $10.21037 /$ cdt.2018.05.08

View this article at: http://dx.doi.org/10.21037/cdt.2018.05.08

Cardiac resynchronization therapy (CRT) nowadays is an important part of invasive heart failure therapy (1). Furthermore, it gains increasing recognition for antibradycardia pacemaker indications with an expected high burden of ventricular pacing by reducing cardiac remodeling (2). With a considerable number of CRT implantations worldwide, the impact of the recent clinical evaluation of wireless endocardial left ventricular (LV) pacing with a novel wireless cardiac resynchronization system (WiSE-CRT, EBR Systems, Sunnyvale, California, USA) by Reddy et al. 2017 should be further debated (3).

Repeatedly reported issues with CRT have been a high non-responder rate at about $30-40 \%$ (4) and suboptimal positioning of the $\mathrm{LV}$ electrodes in the coronary sinus in about $10 \%$ of patients (5). Canine models suggest the superiority of endocardial LV pacing over transvenous epicardial pacing for acute hemodynamic response (6). Atrial and ventricular transseptal approaches for LV endocardial electrode implantation carry the risk of thromboembolism and the need for oral anticoagulation $(7,8)$. In addition, device related infections with potential endocarditis in an already high-risk population of patients with indications for CRT present a considerable burden (9).

In the SELECT-LV Study (Safety and Performance of Electrodes implanted in the Left Ventricle) Reddy et al. included 35 patients ineligible for conventional CRT systems either due to previously failed attempts, an increased risk of the procedure or the "non-responders" to CRT via the coronary sinus. The WiSE-CRT system was implanted in two steps. First an ultrasound transmitter was implanted in a favorable acoustic window as defined by periprocedural echo- and electrocardiography which is then subcutaneously connected to a battery. Step two was the implantation of the $L V$ pacing electrode with a transaortic retrograde approach by anchoring it into the $\mathrm{LV}$ endocardium. The ideal endocardial pacing site was determined by a combination of echo- and electrocardiographic as well as pacing threshold considerations. Antiaggregation/ anticoagulation protocol included dual antiplatelet therapy with aspirin and clopidogrel for three months continued by aspirin thereafter or just a continued oral anticoagulation with warfarin for those with other long-term indications for oral anticoagulation. The WiSE-CRT system requires an implanted conventional pacemaker to receive right ventricular (RV) pacing impulses and transmit impulses to the LV electrode. The set-up of the conventional pacemaker in conjunction with the wireless $\mathrm{LV}$ system is shown in Figure 1.

The success rate of the implantation was 97.1\% (34/35). At 1 month the primary endpoint, a 12-lead ECG showing biventricular pacing, was accomplished in $97.1 \%(33 / 34)$ and in $93.9 \%$ at 6 months $(31 / 33)$. Moderate to marked echocardiographic improvement measured as LV ejection fraction (EF), LV end-systolic volume (ESV) and LV end- 


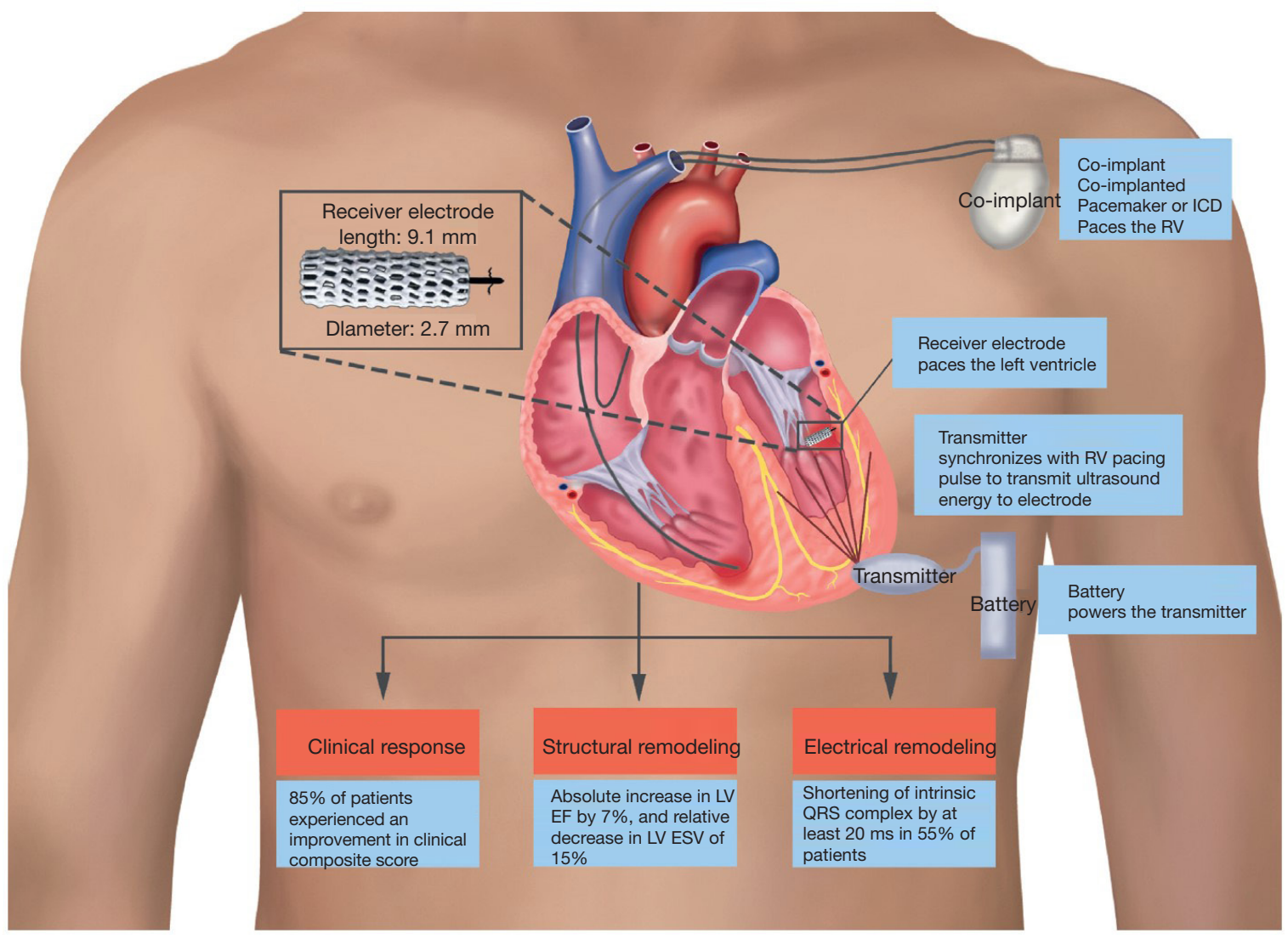

Figure 1 The WiSE-CRT system consists of a transmitter with battery, a receiver electrode in the LV and a co-implanted pacemaker. Up on receiving the RV pacing impulse the transmitter sends an ultrasound signal to the LV electrode. A brief summary of clinical, structural and electrical responses is presented (3). RV, right ventricular; LV, left ventricular; EF, ejection fraction; ESV, end-systolic volume; CRT, cardiac resynchronization therapy; ICD, implantable cardioverter defibrillator.

diastolic volume (EDV) was shown for $70 \%$ at 6 months. Clinical symptoms described by the clinical composite score or the New York Heart Association (NYHA) functional class were reduced and the quality of life improved. Interestingly SELECT-LV compares favorably to the conventional CRT trials. In SELECT-LV 85\% showed an improvement in the clinical composite score compared to $52-69 \%$ in the historical cohorts of MIRACLE ICD, REVERSE and PROSPECT while the rate with a worsening in the score remained lower (3). A direct comparison of the cohorts is impossible due to different patient profiles; however, it remains an interesting aspect to be considered.

In order to evaluate the safety of the system it needs to be brought to attention that the previously introduced WiSE-CRT has been withdrawn due to safety concerns (10). Initially it was intended for 100 patients; however only 17 were enrolled after the occurrence of three periprocedural pericardial effusions, one ending lethally. The delivery system was therefore redesigned to enable atraumatic handling of the LV endocardium. Pericardial effusions have been a concern in RV wireless pacing with the Nanostim ${ }^{\mathrm{TM}}$ and the Micra ${ }^{\mathrm{TM}} \mathrm{TP}$, too $(11,12)$; however this problem has been shown to be reduced in more recent trials $(13,14)$. SELECT-LV showed no occurrence of pericardial effusions. One periprocedural death was reported due to ventricular fibrillation after the delivery catheter got in contact with the $\mathrm{LV}$ endocardium and consequent prolonged resuscitation. Notably an electrode embolization in the tibial artery required no intervention. Two patients developed battery pocket infections, one requiring antibiotic therapy and the other system removal. Two femoral artery complications required a surgical repair. In one patient a stroke occurred three days after the intervention in the context of low level anticoagulation and atrial fibrillation. 
To bring these numbers into context a comparison to other RV leadless pacemakers with more clinical data seems to be appropriate. Overall complication rates of the Nanostim $^{\mathrm{TM}}$ and the Micra ${ }^{\mathrm{TM}}$ TPS have been reported at about $4 \%$ to $6.5 \%$ which is slightly less than $7.5 \%$ for traditional single-lead systems (15). On the contrary the rate of pericardial effusions may be higher for wireless devices in comparison to conventional pacemaker devices $(1.5 \%$ vs. $1.0 \%)(13,15)$. Furthermore, cardiac perforations caused by leadless devices may require a more invasive therapeutic approach due to the larger diameter of the delivery systems (15). For the future, after passing the implantassociated learning curve and with future size-reduction of the delivery systems a further reduction of implantationassociated pericardial effusion can be anticipated. The question still remains whether a safe wireless device could be developed for the thin-walled atria.

Nowadays new therapeutic options are also viewed from a socio-economic perspective. CRT has shown its costeffectiveness compared to medical treatment both with and without implanted defibrillator (16). More recently the cost-effectiveness could also be demonstrated for the patient collective from the REVERSE-study with only mild heart failure symptoms (17). For the WiSE-CRT device a price tag has not yet been set. Considering the two-step implantation approach and for now a lack of competition, the periprocedural and device costs may be higher than that of conventional CRT systems. On contrary in the long-term there could be an economic benefit by lower healthcare utilization costs due to altered complication rates and a high rate of implantation success. This benefit has recently been shown in comparison of quadripolar and bipolar LV coronary sinus leads (18).

There are several uncertainties associated with the wireless stimulation of the $\mathrm{LV}$. The energy transmission via ultrasound is less efficient than via conventional wires. This fact leads to a higher frequency of battery replacements with consequently possible higher risk of implant site infection (19). Self-sustainable devices using a piezoelectric effect as an energy source are currently under development (20). Additionally, it remains unclear what happens if the wireless intracardiac electrodes reach their end of life. In a cadaveric human heart, a maximal number of three Micra ${ }^{\text {TM }}$ TPS devices could have been fitted along the RV septum (21). An extraction of these devices is possible but likely to be complicated due to advanced endothelialization (15).

With the increasing use of quadripolar coronary sinus leads, which can reduce the risk of phrenic nerve stimulation, low pacing thresholds and the need for lead reposition, the number of patients ineligible for conventional LV lead implantation will further decrease (22). Consequently, fewer patients need a wireless device while finding an ideal acoustic window for ultrasound transmission for the wireless devices will probably remain an issue. Considering that SELECT-LV included only patients ineligible for conventional CRT, the patient number for wireless pacing may be reduced.

Technical advancements seem to be very promising for future development. Extending the spectrum of indications from the treatment of bradycardias to the treatment of tachycardias would be revolutionary and has been already evaluated in a first proof of concept pre-clinical study $(22,23)$. Furthermore, the ability of leads to simultaneously sense and pace while exchanging information gathers a great promise (22). Completely different concepts for patients with pacemaker indications are gene therapies to modify non-pacing myocytes to automaticity or application of stem-cell therapy (22). Both approaches are not yet ready for clinical application.

In conclusion, the CRT with wireless LV endocardial pacing and generally all wireless pacing technologies are showing great development with vast potential for further improvements. The effort of further development should definitely be taken to provide the benefits of wireless pacing for a broader patient cohort.

\section{Acknowledgements}

None.

\section{Footnote}

Conflicts of Interest: The authors have no conflicts of interest to declare.

\section{References}

1. Brignole M, Auricchio A, Baron-Esquivias G, et al. 2013 ESC Guidelines on cardiac pacing and cardiac resynchronization therapy: the Task Force on cardiac pacing and resynchronization therapy of the European Society of Cardiology (ESC). Developed in collaboration with the European Heart Rhythm Association (EHRA). Eur Heart J 2013;34:2281-329.

2. Curtis AB, Worley SJ, Adamson PB, et al. Biventricular 
Pacing for Atrioventricular Block and Systolic

Dysfunction. N Engl J Med 2013;368:1585-93.

3. Reddy VY, Miller MA, Neuzil P, et al. Cardiac Resynchronization Therapy With Wireless Left Ventricular Endocardial Pacing: The SELECT-LV Study. J Am Coll Cardiol 2017;69:2119-29.

4. McAlister FA, Ezekowitz J, Hooton N, et al. Cardiac Resynchronization Therapy for Patients With Left Ventricular Systolic Dysfunction: A Systematic Review. JAMA 2007;297:2502-14.

5. Spragg DD, Dong J, Fetics BJ, et al. Optimal Left Ventricular Endocardial Pacing Sites for Cardiac Resynchronization Therapy in Patients With Ischemic Cardiomyopathy. J Am Coll Cardiol 2010;56:774-81.

6. Bordachar P, Grenz N, Jais P, et al. Left ventricular endocardial or triventricular pacing to optimize cardiac resynchronization therapy in a chronic canine model of ischemic heart failure. Am J Physiol Heart Circ Physiol 2012;303:H207-15.

7. van Gelder BM, Scheffer MG, Meijer A, et al. Transseptal endocardial left ventricular pacing: An alternative technique for coronary sinus lead placement in cardiac resynchronization therapy. Heart Rhythm 2007;4:454-60.

8. Betts TR, Gamble JH, Khiani R, et al. Development of a Technique for Left Ventricular Endocardial Pacing via Puncture of the Interventricular Septum. Circ Arrhythm Electrophysiol 2014;7:17-22.

9. Klug D, Balde M, Pavin D, et al. Risk factors related to infections of implanted pacemakers and cardioverterdefibrillators: results of a large prospective study. Circulation 2007;116:1349-55.

10. Auricchio A, Delnoy PP, Butter C, et al. Feasibility, safety, and short-term outcome of leadless ultrasoundbased endocardial left ventricular resynchronization in heart failure patients: results of the Wireless Stimulation Endocardially for CRT (WiSE-CRT) study. Europace 2014;16:681-8.

11. Reddy VY, Knops RE, Sperzel J, et al. Permanent Leadless Cardiac Pacing: Results of the LEADLESS Trial. Circulation 2014;129:1466-71.

Cite this article as: Holubec T, Beckers J, Holubcova Z, Walter V, Walther T. Cardiac resynchronization therapy with wireless left ventricular endocardial pacing: is this the direction to go? Cardiovasc Diagn Ther 2018;8(4):534-537. doi: 10.21037/ cdt.2018.05.08
12. Bonner M, Eggen M, Haddad T, et al. Early Performance and Safety of the Micra Transcatheter Pacemaker in Pigs. Pacing Clin Electrophysiol 2015;38:1248-59.

13. Reddy VY, Exner DV, Cantillon DJ, et al. Percutaneous Implantation of an Entirely Intracardiac Leadless Pacemaker. N Engl J Med 2015;373:1125-35.

14. Roberts PR, Clementy N, Al Samadi F, et al. A leadless pacemaker in the real-world setting: The Micra Transcatheter Pacing System Post-Approval Registry. Heart Rhythm 2017;14:1375-9.

15. El-Chami MF, Merchant FM, Leon AR. Leadless Pacemakers. Am J Cardiol 2017;119:145-8.

16. Yao G, Freemantle N, Calvert MJ, et al. The long-term cost-effectiveness of cardiac resynchronization therapy with or without an implantable cardioverter-defibrillator. Eur Heart J 2007;28:42-51.

17. Gold MR, Padhiar A, Mealing S, et al. Economic Value and Cost-Effectiveness of Cardiac Resynchronization Therapy Among Patients With Mild Heart Failure: Projections From the REVERSE Long-Term Follow-Up. JACC Heart Fail 2017;5:204-12.

18. Behar JM, Chin HM, Fearn S, et al. Cost-Effectiveness Analysis of Quadripolar Versus Bipolar Left Ventricular Leads for Cardiac Resynchronization Defibrillator Therapy in a Large, Multicenter UK Registry. JACC Clin Electrophysiol 2017;3:107-16.

19. Benditt DG, Goldstein M, Belalcazar A. The leadless ultrasonic pacemaker: a sound idea? Heart Rhythm 2009;6:749-51.

20. Marbán E, Cho HC. Biological pacemakers as a therapy for cardiac arrhythmias. Curr Opin Cardiol 2008;23:46-54.

21. Omdahl P, Eggen MD, Bonner MD, et al. Right Ventricular Anatomy Can Accommodate Multiple Micra Transcatheter Pacemakers. Pacing Clin Electrophysiol 2016;39:393-7.

22. Sperzel J, Burri H, Gras D, et al. State of the art of leadless pacing. Europace 2015;17:1508-13.

23. Tjong FV, Reddy VY. Permanent Leadless Cardiac Pacemaker Therapy: A Comprehensive Review. Circulation 2017;135:1458-70. 